\title{
Enjeux de la traduction du roman caribéen plurilingue. Étude de traductions de Daniel Maximin, Ernest Pépin et Maryse Condé
}

\section{Challenges Represented by the Translation of Plurilingual Caribbean Novels.}

\section{A Study of Translations of Daniel Maximin, Ernest Pépin and Maryse Condé Works}

Anaïs Stampfli [Anais.stampfli@unil.ch]

Université de Lausanne, Suisse

\section{RÉSUMÉ}

Les traducteurs de romans caribéens sont confrontés à la délicate question de la transposition d'un contenu plurilingue. Ils ont pour tâche de rendre accessible à un public éloigné une œuvre décrivant un univers référentiel multiple tant sur le plan culturel que linguistique. Nous observerons ici les complications inhérentes à la traduction de romans de trois auteurs guadeloupéens contemporains : Daniel Maximin, Ernest Pépin et Maryse Condé. Nous analyserons également les différentes stratégies mises en place par les traducteurs pour transposer au mieux l'univers dépeint par ces trois romanciers. Il s’agira ainsi de déterminer les enjeux, les possibles et les limites de la traduction de romans plurilingues caribéens.

\section{MotS CLEFS}

Traduction ; roman caribéen ; plurilinguisme ; opacité ; paratexte ; interprétation du traducteur

\begin{abstract}
The translators of Caribbean novels have to deal with the delicate question of the transposition of multilingual content. Their task is to make accessible to a distant audience a work describing a multiple referential universe, both culturally and linguistically. In this article, I will observe the complications implied by translating novels by three contemporary Guadeloupean authors: Daniel Maximin, Ernest Pépin and Maryse Condé. I will also analyze the different strategies adopted by the translators to transpose as well as possible the universe depicted by these three novel writers. The purpose will be to determine the stakes, the possibilities and the limits of the translation of plurilingual Caribbean novels.
\end{abstract}

\section{KEYWORDS}

Translation; Caribbean novel; multilingualism; opacity; paratext; translator interpretation

REÇU 2020-11-18; ACCEPTE 2021-01-20 
Traduire un roman caribéen représente un défi de taille. Ces œuvres s’inscrivent dans un cadre plurilingue à l'image de la situation linguistique des Antilles et elles sont empreintes d'un univers référentiel culturel créole souvent inconnu du lecteur du texte cible. Il revient alors au traducteur de trouver une solution pour faire entendre toutes les subtilités langagières et culturelles du texte source sans dénaturer l’ouvre ni entraver laccès au sens. Nous nous intéresserons ici aux stratégies auxquelles ont eu recours trois traducteurs : Clarisse Zimra et Richard Philcox qui ont respectivement traduit Daniel Maximin et Maryse Condé en anglais ainsi que Marie-José Hoyet qui a traduit Ernest Pépin en italien. Ces trois romanciers guadeloupéens ont pour point commun de présenter une écriture plurilingue mêlant au français des expressions issues du créoles mais aussi d’autres langues caribéennes comme l'espagnol et langlais. Daniel Maximin et Ernest Pépin sont également poètes. Leur créativité poétique se retrouve dans leurs œuvres romanesques où il est fréquent de retrouver des jeux sur les sonorités, sur le rythme ainsi que des jeux de mots plurilingues. La tâche du traducteur se retrouve ainsi des plus ardues. Il semble confronté à une alternative : se faire à son tour créateur en séloignant du texte source pour trouver des expressions suggestives équivalentes dans la langue cible ou refuser de traduire en expliquant dans le paratexte le sens des passages non-traduits. Il s’agira ainsi d'explorer les différentes stratégies mises en œuvre pour relever le défi de la traduction de romans caribéens plurilingues.

\section{Daniel Maximin : L'Isolé soleil, version anglophone}

L'Isolé soleil est le premier volet d'une trilogie caribéenne complétée par Soufrières et L'T̂le et une nuit. Avec ces trois romans, Daniel Maximin donne à voir une quête identitaire appuyée sur une approche géopoétique ${ }^{1}$ de l'espace caribéen. Clarisse Zimra, la traductrice de L'Isolé soleil accompagne le texte cible d'une présentation détaillée de l’ouvre de Daniel Maximin (Daniel Maximin 1989: XXXVIII). Les commentaires accompagnant l'acte de traduction sont très développés. Elle introduit le roman par un glossaire (Daniel Maximin 1989 : IX-X) expliquant une vingtaine de termes créoles (bakoua, soubarou...), de termes français spécifiques (comme l'acronyme CRS) ou encore de noms de personnages qui jouent un rôle central dans les contes folkloriques créoles (Armored Fish : Poisson-Armé, colibri...). Elle propose également en appendice (Daniel Maximin 1989 : 289) une traduction des prières et chants qui ne sont présentés qu’en créole dans le roman de Daniel Maximin. Enfin, Clarisse Zimra propose une introduction d'une cinquantaine de pages dans laquelle sont présents son analyse du roman, une explication du contexte historique et des influences littéraires, l'extrait d'un entretien avec l'auteur ainsi qu'une explication des difficultés qu'il y a à traduire une telle œuvre :

The richness of the language presents a challenge to the uninitiated reader and, more importantly perhaps, to translators faced with puns in French, English - and sometimes a curious private mixture of Italo-Spanish - and the rhythmic and thematic influence of black music, in particular, Afro-American and Afro-Cuban jazz. ${ }^{2}$ (Daniel Maximin 1989 : XI).

1 L'idée étant avec l'approche géopoétique telle que l'a conçue Kenneth White de penser le rapport entre l'Homme et la Terre. Daniel Maximin a également consacré un essai à ce sujet (Daniel Maximin 2006).

2 (La richesse de la langue présente un défi au lecteur non initié et, plus important encore peut-être, aux traducteurs qui doivent faire face aux jeux de mots en français, en anglais - et parfois à un curieux mélange propre à l'auteur d’italo-espagnol - et à l’influence rythmique et thématique de la musique noire, particulièrement du jazz Afro-Américain et Afro-Cubain), (je traduis). 
Le défi réside également dans le fait que l’auteur, avec ses alliances de voix surprenantes, a voulu dérouter le lecteur, il ne s'agit donc pas d’aller vers une trop grande transparence monolingue qui aplatirait l'effet de surprise :

This multivoiced narrative strategy carries over the linguistic registers in order further to decenter the exploded writing self. The full impact of the original depends a good deal on what I would call a systematic "surprise effect" - grammatical as well as semantic - the better to remind its French readers that Creole, too, is a foreign language not entirely assimilable to French cultural codes. This makes translating Maximin a nearly impossible task [...] (Daniel Maximin 1989 : LVII-LVIII) ${ }^{3}$.

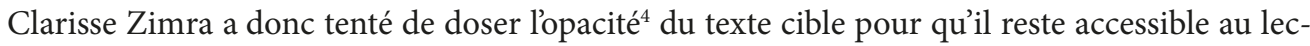
teur anglophone : " In Maximin's case, the problem then becomes how to maintain an acceptable degree of opacity for the English or American public, compatible to, but obviously not always exactly similar to, the opacity experienced by the French public. $»^{5}$ (Daniel Maximin 1989 : LVIII). Elle présente donc des explications détachées du texte (situées dans le glossaire initial ou dans l’appendice final) pour que le lecteur dérouté par l'étrangeté du texte puisse, s’il le souhaite, chercher à en savoir plus sur les langues mêlées par Daniel Maximin :

In order for the present translation to retain this playful yet frustrating density, and at Maximin's urgent request, all of the original Creole passages have been kept with a little editorial interference as possibe (although, occasionally, a discrete contextual gloss is provided). Readers who do not wish to play cat and mouse may refer to the glossary or the appendix ${ }^{6}$. (Daniel Maximin 1989: LXI).

Cette stratégie semble être un compromis permettant de découvrir dans un premier temps les langues du texte telles quelles tout en ayant ensuite accès, si nécessaire, à la traduction. La retranscription des chants en appendice final permet ainsi de faire entendre dans un premier temps les paroles en créole. En faisant ce choix, la traductrice respecte l’attention que Daniel Maximin porte à la musicalité.

Dans le chapitre « Onze proverbes », l'auteur retranscrit sous une graphie distinguée (en lettres majuscules) onze proverbes créoles qui sont présentés en « français calqué » ${ }^{7}$ pour faire entendre

3 [Cette stratégie narrative à plusieurs voix joue avec les registres linguistiques pour décentrer, éclater lécriture de soi. L'impact d’ensemble de loriginal dépend beaucoup de ce que jappellerais un système d' " effet de surprise » - aussi bien grammatical que sémantique - pour mieux rappeler aux lecteurs français que le créole est aussi une langue étrangère non entièrement assimilable aux codes culturels français. Ceci fait de la traduction de Maximin une tâche presque impossible.] (Je traduis).

4 On ne peut employer ce terme sans penser au concept d'Édouard Glissant qui revendique avec l'Opacité un droit à : « l'affirmation et la subsistance d'une singularité non réductible à un modèle universel » (Christine Ndiaye 2005: 182).

5 [Dans le cas de Maximin, le problème devient alors de savoir comment maintenir un degré acceptable d’opacité pour le public anglais ou américain, compatible, bien quévidemment pas toujours exactement semblable, à l'opacité expérimentée par le public français.] (Je traduis).

6 [Pour que la présente traduction conserve cette densité espiègle bien qu'irritante et à la demande urgente de Maximin, tous les passages originaux en créole ont été gardés avec aussi peu d'interférence éditoriale que possible (bien que, de temps en temps, on fournisse une explication contextuelle discrète). Les lecteurs qui ne veulent pas jouer au chat et à la souris peuvent se référer au glossaire ou à l'annexe.] (Je traduis).

7 Il s'agit d'une traduction mot à mot qui respecte en français la syntaxe créole pour faire entendre une autre langue (le créole ici) derrière la formulation française inédite. 
la langue autre derrière ces formules inédites. Clarisse Zimra a saisi le sens de la démarche de Daniel Maximin (" the eleven proverbs were given in French, but with the original Creole syntax. The result is that they may look odd, even sound archaic, but are nonetheless intelligible " (Daniel Maximin 1989 : LVIII) ${ }^{8}$. Elle a donc veillé à ne pas gommer cette étrangeté en transposant les proverbes en un anglais énigmatique. Clarisse Zimra traduit : «LE PREMIER PROVERBE DIT : LÀ OÙ IL Y A DES OS, LÀ OÜ IL Y A DES CHIENS... » (Daniel Maximin 1981 : 45) par « THE FIRST PROVERB SAYS : WHERE THERE ARE BONES, WHERE THERE ARE DOGS...) (Daniel Maximin 1989 : 40). La répétition de la conjonction "where" altère la structure usuelle de la phrase et laisse entendre qu'il s'agit là d'un calque du créole.

Par endroits, les jeux de mots de Daniel Maximin sont fondés sur des termes polysémiques en français, mais pas en anglais. La traductrice se voit donc contrainte d'accoler le terme source à sa traduction. La formule :
Je,
$t u$,
île
aile

Il faut faire avec ce que l'on EST (Daniel Maximin 1981 : 99)

Devient ainsi :

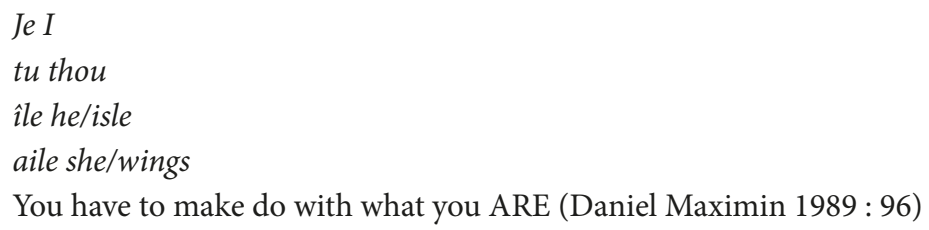

Lorsque c'est possible, la traductrice tente tout de même de trouver un équivalent au même son ( $t u$ » et «thou») et au même sens (« île» et « isle»). Cependant, il lui est nécessaire de rappeler la langue source, car dans ces cas une traduction sans perte de sens est impossible. Clarisse Zimra ajoute ainsi à la dimension plurilingue de l’œuvre en laissant apparentes langue source et langue cible :

Je voulais être SOLEIL

J'ai joué avec les mots

J’ai trouvé L'ISOLÉ (Daniel Maximin $1981:$ 105)

Devient :

I wanted to be SOLEIL/SUN

I played with words.

I found ISOLE/LONE (Daniel Maximin 1989 : 102).

8 [Les onze proverbes ont été donnés en français, mais avec la syntaxe créole originale. Il en résulte des formules qui peuvent paraître étranges, voire archaïques, mais elles restent malgré tout intelligibles.] (Je traduis). 
La traduction conserve également les couplets espagnols de la version originale. Elle propose cependant une légère modification dans sa transcription : Clarisse Zimra note en introduction que Daniel Maximin crée « sometimes a curious private mixture of Italo-Spanish » (Daniel Maximin $1989: 11)^{9}$. De fait, certains termes espagnols sont accentués à l'italienne. Le romancier guadeloupéen altère l'accentuation classique espagnole. Peut-être cette altération vise-t-elle à moduler le rythme des paroles citées. La traductrice voit plutôt ces ajouts comme une faute qu'elle corrige dans la version anglophone où " púedes » (Daniel Maximin 1981 : 133) devient "puedes" (Daniel Maximin 1989 : 131) « asi » (Daniel Maximin 1981 : 134) devient « así » (Daniel Maximin 1989 : 131) et « qué » (Daniel Maximin $1981:$ 134) devient « que» (Daniel Maximin $1989: 131$ ).

L'Isolé soleil est un roman riche en références intertextuelles. La traductrice tente d'en restituer le plus possible, mais certaines sont légèrement effacées. C’est le cas du titre de chapitre " "E" comme Capitale de la douleur » (Daniel Maximin 1981 : 132) qui se réfère explicitement au recueil de Paul Éluard et a été traduit non pas par Capital of pain, mais par " "E" as in Birthplace of pain " (Daniel Maximin 1989 : 130).

Hormis ces quelques aménagements de Clarisse Zimra, la traduction anglaise du roman de Daniel Maximin restitue telles quelles les voix mêlées de L'Isolé soleil tout en essayant de donner au lecteur anglophone accès à l'univers dépeint par l'auteur.

\section{Ernest Pépin : L'Homme-au-Bâton, traduction italophone de Marie-José Hoyet}

Avec L'Homme-au-Bâton, Ernest Pépin donne vie à la figure légendaire de l'incube antillais. La version italophone de L'Homme-au-Bâton fidèlement intitulée L'Uomo col bastone a été publiée aux éditions Lavoro à Rome en 1996 soit quatre ans après la publication originale. En quatrième de couverture, le lecteur peut découvrir un résumé de l’œuvre ainsi qu'une petite biographie de l'auteur précisant qu'il a gagné en 1994 le Prix des Caraïbes pour L’Homme-au-Bâton qui est sa première œuvre traduite en italien. Marie-José Hoyet qui a également traduit le recueil poétique Africa solo d'Ernest Pépin en 2003 propose au lecteur italophone une introduction sur le contexte du roman ainsi que sa structure et sur le parcours de l'auteur. La traductrice connait bien le contexte culturel caribéen et plus largement latino-américain. Elle le prouve en proposant de judicieux parallèles. Marie-José Hoyet explique par exemple dans son introduction qu'Ernest Pépin se plait à faire le portrait d'une myriade de personnages du Pointe-à-Pitre des années 1950 à la manière du Trinidadien Vidiadhar Surajprasad Naipaul et du Brésilien Jorge Amado : « Allo stesso modo di Naipaul nella Trinidad di Miguel Street o di Amado nella Bahia de La Bottega dei miracoli, Pépin attinge da questo microcosmo del Caribe una galleria di ritratti che riflette tutta la gamma delle tipologie umane di un mondo ormai scomparso, in cui davvero "tutto può succedere" " (Ernest Pépin 1996 : VIII) ${ }^{10}$. L'expression « tutto può succedere » est, soit dit en passant, reprise et traduite

9 [Il crée parfois un mélange étrange et tout personnel d'italo-espagnol] (Je traduis).

10 [De la même manière que Naipaul dans le Trinidad de San Miguel Street ou qu'Amado dans le Bahia de La Boutique des miracles, Pépin puise dans ce microcosme caribéen une galerie de portraits qui reflète toute la gamme des typologies humaines d'un monde maintenant disparu dans lequel vraiment « tout peut arriver »] (Je traduis). 
des dernières pages de Pluie et vent sur Télumée Miracle ${ }^{11}$, de Simone Schwarz-Bart. La traductrice se réfère à plusieurs reprises à la romancière guadeloupéenne notamment pour évoquer son traitement de la folie insulaire, qui d’après elle, a inspiré Ernest Pépin.

Marie-José Hoyet a ainsi tenté au mieux de donner à lire cet univers caribéen au lecteur italophone. Elle mêle aux quelques notes de bas de page de lauteur ses propres notes expliquant des termes créoles laissés tels quels dans l’ouvre source. Ces termes sont mis en italiques. Ce faisant, elle met en relief leur étrangeté. C’est le cas de rara (Ernest Pépin 1996 : 7), et tafia (Ernest Pépin 1996 : 8). Le terme « chabine " (Ernest Pépin 1992 : 13), annoté par l'écrivain, mais non distingué typographiquement, est aussi mis en italiques par la traductrice par souci d'uniformisation. Ses notes et celles d'Ernest Pépin sont ainsi harmonisées. Contrairement aux traducteurs anglophones, Rose-Myriam Réjouis et Val Vinokur ${ }^{12}$, Marie-José Hoyet ne semble pas tenir à distinguer ses propres notes de celles d'Ernest Pépin. Elle complète les notes de lécrivain guadeloupéen pour faciliter la lecture du texte cible. L’entité divine «maliémin » (Ernest Pépin $1992: 41$ ) qui nest pas définie dans l'œuvre source (et qui peut sembler tout aussi mystérieuse au lecteur francophone qu'au lecteur italophone) est ainsi désignée en note par la traductrice comme une "deformazione di Maria amata (in francese : Marie aimée). Ceremonia religiosa di origine induista " (Ernest Pépin $1996: 28)^{13}$. En ajoutant des explications, la traductrice prend la liberté de réduire lopacité du texte source. Tous les termes annotés sont répertoriés en un glossaire final pour que le lecteur prenne la mesure de la plurilinguicité du texte source.

Toutefois, il arrive que dans la version de Marie-José Hoyet certaines expressions plurilingues ou issues de la culture caribéenne soient passées sous silence. Tandis que dans l’ouvre source TiSaint-Louis «était arrivé avec un vivaneau bien frais» (Ernest Pépin $1992: 111$ ), « si era presentato con un pesce freschissimo " (Ernest Pépin 1996 : 75) dans la version traduite. Autrement dit, le nom du poisson tropical est standardisé en un "poisson très frais ». De même, au terme créole « bobo » (Ernest Pépin 1992:17), désignant une prostituée, est préférée l’expression italienne « sgualdrina » (Ernest Pépin 1996 : 13) signifiant "garce". On peut se demander s'il s’agit ici d’un phénomène de censure ou plus simplement (et plus probablement) d'une volonté de simplification du passage contenant déjà plusieurs termes créoles expliqués en note de bas de page. Certaines occurrences de français régional des Antilles sont également passées sous silence, la particularité de ladverbe «bien souventement » (Ernest Pépin 1992 : 22) n’est par exemple pas rendue dans la traduction qui utilise le terme commun «spesso » (Ernest Pépin 1996: 82). Si ces modifications peuvent être vues comme des omissions ou des choix de simplification, il y a d'autres pertes visà-vis de l’ouvre source qui sont dues à la dimension poétique de la langue d’Ernest Pépin, difficilement traduisible.

En effet, tout comme Daniel Maximin, Ernest Pépin est avant tout poète et aime jouer avec les sons et avec la parenté phonique de certaines expressions françaises et créoles. Lorsqu'un personnage sécrie « Bon Dieu, mi bel bitin, quel beau butin de femme » (Ernest Pépin $1992: 16$ ), l’auteur

11 «Tant que le soleil n’est pas couché, tout peut arriver 》 (Simone Schwarz-Bart 1972 : 246).

12 Ces traducteurs des romans Solibo Magnifique et Texaco de Patrick Chamoiseau ont ajouté aux notes de l'auteur leur propres notes de bas de page qu'ils ont pris soin de présenter entre crochets.

13 Nous avons trouvé ailleurs que ce terme ne serait pas d’origine française : selon Laënnec Hurbon, "maliémin" serait une déformation créole « de Mariamman, divinité indoue du Sud de l'Inde connue comme divinité de la variole » (Laënnec Hurbon $2000: 314$ ). 
joue sur la parenté sonore des termes « bitin » (signifiant « chose » en créole) et «butin ». Il s'agit là d'une association poétique franco-créole difficile à rendre en langue italienne qui na pas connu de phénomène de créolisation (bien quétant dans une relation forte avec des dialectes régionaux encore bien vivants). Faute de restituer le jeu sonore, la traductrice a tout de même tenté de restituer le sens avec l'expression «Dio buono, mi bel bitin ${ }^{14}$, che bel pezzo di donna » (Ernest Pépin 1996 : 9). Ici l'expression "pezzo di donna" signifiant "bout de femme" restitue l'esprit de la réplique.

Lorsque les romans antillais présentent une forte teneur poétique, certaines pertes semblent inévitables dans la traduction, comme constaté lors de létude de la transposition de L'Isolé soleil de Daniel Maximin. Cependant, la traductrice n’a pas abandonné le projet et, forte de son bagage culturel caribéen, elle a pris ici le parti de rendre sa traduction la plus accessible possible au lecteur italophone.

À défaut d'éléments témoignant précisément de la réception des traductions d'Ernest Pépin en Italie, nous pouvons nous référer à l'étude menée en 2007 par Carla Fratta et Francesca Torchi. Ces chercheuses de l'Université de Bologne constatent, à l'inverse de l'observation faite par la traductrice Anna Devoto en $2002^{15}$, un intérêt grandissant pour les littératures caribéennes : « De façon générale, l'intérêt pour ces nouvelles littératures [caribéennes] [...] augmente de jour en jour en Italie, un phénomène qui est souligné par la récente publication de volumes qui visent à diffuser la culture et/ou littérature de la Caraïbe auprès d'un public plus vaste [...] » (Carla Fratta et Francesca Torchi 2007 : 332). Nous pouvons retenir de la liste faite par Carla Fratta et Francesca Torchi le recueil Voci dalle isole. Narrativa delle aree insulari francofone de Marie-Josée Hoyet. Les universitaires précisent que « les auteurs de ces ouvrages visent à réveiller l'intérêt du public italien et d'un point de vue historique et culturel et d'un point de vue littéraire et linguistique. Un long dossier de Nigrizia rédigé par Marie-Josée Hoyet confirme cet intérêt croissant " (Carla Fratta et Francesca Torchi 2007 : 332).

\section{Maryse Condé : Traversée de la Mangrove, version anglophone traduite par Richard Philcox}

Avec Traversée de la Mangrove, Maryse Condé propose un tableau de la société guadeloupéenne à partir de la venue d'un mystérieux étranger, Francis Sancher. La version anglophone de Traversée de la Mangrove a été publiée en 1995 aux éditions newyorkaises First Anchor Book. La traduction de Ségou en 1987 par Barbara Bray avait déjà permis de faire découvrir Maryse Condé au lectorat anglophone. L'écrivaine jouit depuis lors d'un succès américain assurant la traduction de presque chacun de ses livres. Les éditeurs de Crossing the mangrove ont à ce sujet vu le succès de Segu comme un argument de vente et rappelé en couverture du roman que Maryse Condé est aussi

14 Il s'agit là d'une des rares expressions créoles mises en italique par la traductrice sans qu'elle ne propose de traduction. Elle a peut-être voulu faire comprendre par-là que c'est du créole mais le sens de l'expression importe ici moins que les sons...

15 Anna Devoto affirme à propos de sa traduction italienne du roman Ravines du Devant-jour de Raphaël Confiant : « Malheureusement, cela n’a eu aucun succès. En Italie, après les années 1960 très politisées, on s'est beaucoup intéressé à la Négritude. Les choses ont beaucoup changé. On ne savait plus ce quétait l'indiennité, la créolité. Tout était très nouveau. Les livres que j’ai traduits ont été tirés à environ mille cinq cents exemplaires et vendus à cinq cents : une catastrophe. Cet éditeur qui avait fait d’autres livres africains, indiens ou arabes a fermé ses portes. » (Anna Devoto 2003 : 27). 
"Author of Segu ». La biobibliographie de la romancière est d'ailleurs rappelée en fin d'ouvrage ainsi qu'en quatrième de couverture. Cette quatrième de couverture introduit le roman comme étant un "Rashomon-like novel ». Léditeur fait appel à une référence japonaise ${ }^{16}$ pour donner un aperçu sur ce roman multi-focal. On aurait peut-être pu penser à une référence plus proche géographiquement comme la Chronique d’une mort annoncée de Gabriel García Marquez, rassemblant différents points de vue sur le meurtre de Santiago Nasar. Cependant, la pluri-focalisation est une technique d'écriture universelle et les éditeurs, en choisissant cet exemple, ont peut-être voulu resituer la démarche de Maryse Condé dans un contexte plus vaste. Richard Philcox, le traducteur et époux de la romancière, a fait de même en affirmant sêtre appuyé sur les romans de Virginia Woolf pour traduire Traversée de la Mangrove. Il explique en préface avoir évité des références trop proches du contexte géographique du roman par peur de le dénaturer en le transposant dans une île voisine anglophone à l'identité totalement différente. C’est pour cette raison qu'à l'inverse d'Evelin Van Hemert traduisant Patrick Chamoiseau ${ }^{17}$, il a choisi de ne pas traduire les expressions créoles à base lexicale francophone en créole à base lexicale anglophone : «I could have researched the English-speaking West Indian equivalents of many Creole expressions, but this would have distanced the reader from the French and Creole-speaking environment of Guadeloupe and transported him or her to Barbados or Jamaica (Maryse Condé 1995 : VIII) ${ }^{18}$. Il n’a pas non plus voulu créer de néologisme ${ }^{19}$ car il juge cette démarche indissociable d'un geste idéologique de conquête de la langue : «I could have invented words in English (I did in one or two cases) but I (and even less the author) have no quarrel with the English language on the same level as Creole quibbles with French» (Maryse Condé 1995 : VIII) ${ }^{20}$. Le traducteur a donc pris la liberté de se distancer quelque peu du texte source ${ }^{21}$ pour se concentrer sur la transmission des tonalités de l'œuvre qui, à ses yeux, sont essentielles au roman : «I decided to concentrate on the

16 Rashomon est un film de 1950 reprenant la nouvelle éponyme de Ruyunosuke Akutagawa (1915) relatant quatre versions très différentes d'un seul et même crime.

17 Dans sa traduction néerlandaise de L'Esclave vieil homme et le molosse, Evelin Van Hemert a fait le choix d'une traduction conservant le plurilinguisme de l’œuvre source en alternant le néerlandais et le créole néerlandophone surinamien, le sranan.

18 [J'aurais pu chercher l'équivalent caribéen anglophone de beaucoup d’expressions créoles, mais ça aurait éloigné le lecteur de l'environnement franco-créolophone de la Guadeloupe en le ou la projetant à la Barbade ou en Jamaïque]. (Je traduis).

19 Ce choix du néologisme a été fait par la traductrice de Chamoiseau en allemand, Giovanna Waeckerlin Induni. À ce sujet, voir Stampfli, A. (2014).

20 [J'aurais pu inventer des mots en anglais (je l'ai fait dans un ou deux cas) mais je (et encore moins l'auteur) n'ai aucun compte à régler avec la langue anglaise à la manière du créole chicanant le français.] (Je traduis).

21 On remarque cette distanciation en observant la liberté prise vis-à-vis des notes de bas de page : Certaines notes de bas de pages sont ajoutées pour rendre l'œuvre accessible au lecteur non-francophone. C'est le cas de la note au bas de la page 8 précisant l'origine et la nature du bateau L’Aurélie. Le traducteur procède également à l'inverse à la dissimulation de certaines notes de bas de page. Certains termes en français régional et en créole tels que " la trace ${ }^{1}$ " ou " pié-chans ${ }^{3}$ » (Maryse Condé 1989 : 14) sont expliqués en note de bas de page dans le roman de Maryse Condé («1. Chemin de forêt », « 3. Lianes parasites » (Maryse Condé 1989: 14) et dans la version anglophone ils sont simplement rendus par les traductions anglaises « forest path » et «creepers " (Maryse Condé 1995 : 2). Ce détachement qui se retrouve même dans l'évocation de surnoms et noms communs : "Moïse dit Maringoin ${ }^{1}$ " (1. Moustique) (Maryse Condé 1989 : 18) devient « Moïse nicknamed Mosquito ( (Maryse Condé 1995 : 5).

Cet effacement de la tierce langue marque le refus de trouver un équivalent en créole anglophone qui serait dénaturant mais de ce fait il réduit également un peu ce qui fait la particularité du roman de Maryse Condé : la dimension plurilinguistique. 
tone and register of these voices speaking from this wake ceremony and talking to us, even chatting to us, as we turn the pages » (Maryse Condé 1995 : VIII) ${ }^{22}$. Pour introduire les différentes voix de Traversée de la Mangrove, Richard Philcox s'est ainsi inspiré des mouvements des romans de Virginia Woolf et en particulier Promenade au phare. Il explique :

I found that tone and register of voice, those trifling details with universal significance, the way the colors of Nature interweave in personal lives and the way the reader is made to look at the horizon and then back again to himself or herself. I found all this in Virginia Woolf, and in particular in her novel To the Lighthouse. Her stream of consciousness technique spoke in and out of the characters' lives and talks to us in a voice that touches the right cord. You might be wondering how the register for an English middle-class family could be appropriate for the inhabitants of a small village in Guadeloupe. It is because I sense a similarity of purpose and a mastery of style in both authors that transcends the two very different contexts of a holiday home on the English coast and a tropical village. (Maryse Condé 1995 : VIII-IX) ${ }^{23}$.

Richard Philcox évoque ici une intuition de lecture toute personnelle ("I sense ») qui le pousse à rapprocher Maryse Condé de Virginia Woolf au nom des monologues intérieurs exprimant de semblables divagations spontanées au gré des pensées des personnages. Il est vrai qu’en découvrant les pensées de Léocadie Timothée, on peut voir quelque chose de woolfien dans l'écriture de Maryse Condé :

Quand je me réveillai le lendemain matin, je me regardais dans ma glace et je me vis encore plus laide, encore plus noire avec une expression que je ne me connaissais pas : un air méchant et dur, fermé comme une porte de prison. Je compris que j’étais devenue une autre femme. Sans amour, le cœur des femmes se durcit. Il devient une savane désolée où ne poussent que les cactus. À partir de ce moment-là, des histoires encore plus terribles commencèrent à circuler sur mon compte. Les gens dirent que je faisais des kakwè ${ }^{1}$ (1. Sortilèges). Ils se mirent à mappeler ouvertement « vié vo$\operatorname{lan}^{2} »(2$. Vieille sorcière), « vié Satan » (Maryse Condé 1989: 147).

Ici, le personnage se laisse porter par ses pensées sombres et se découvre au fil de ces mêmes pensées qui la caractérisent et influent sur son être et son paraître. Le personnage ne se « connaissai $[t]$ » plus et le cours de ses pensées qui prennent le contrôle sur sa personne va l'aider à « compr[endre] » ce qu'elle est devenue, comme l'héroïne de Promenade au phare.

22 [J'ai décidé de me concentrer sur le ton et le registre de ces voix émises lors de la veillée et s'adressant à nous, bavardant même avec nous au fil des pages que l'on tourne.] (Je traduis).

23 [J'ai trouvé ce ton et ce registre de voix, ces détails insignifiants à signification universelle, la manière dont les couleurs de la nature interfèrent dans les vies personnelles et la manière dont le lecteur est voué à regarder vers l'horizon puis à nouveau derrière elle ou lui. J'ai trouvé tout cela chez Virginia Woolf, et en particulier dans son roman Promenade au phare. Sa technique de courant de conscience évoque d'un point de vue externe et interne les vies des personnages et nous parle d'une voix qui vise juste. Vous pourriez vous demander comment le registre d'une famille de classe moyenne anglaise pourrait être approprié pour évoquer les habitants d'un petit village guadeloupéen. C’est parce que je ressens une proximité dans l'objectif et la maîtrise du style chez ces deux auteures qui transcendent les deux contextes très différents de la maison de vacance sur la côte anglaise et du village tropical.] (Je traduis). 
À la lecture de ce passage, on comprend qu'en traduisant Richard Philcox avait le style de Virginia Woolf en tête :

When I awoke the next morning, I looked at myself in the mirror and saw myself even uglier, even blacker, together with an expression I had never seen before: a hard, mean look, shut tight as a prison door. I realized I had become another woman. Without love, a woman's heart hardens. It becomes a desolate savanna where only cacti grow. From that moment on even worse stories started to circulate about me. People said I vast kakwè ${ }^{31}$ (31 Spells). They began to call me openly "vié volan"32 (32 Old witch) and "old devil". (Maryse Condé 1995 : 119).

Sa première phrase ample reprend celle de Maryse Condé mais elle fait également penser à celles de Virginia Woolf où les phrases semblent s'engendrer d’elles-mêmes au fil du flot de pensées exprimées librement dans le cadre du stream of consciousness.

Cependant, le roman de Maryse Condé ne fait pas que livrer des intériorités. Au contraire, il est dans l'interaction constante entre les pensées des personnages et les propos de leur entourage. Le passage cité ci-dessus illustre bien ce dialogue : les pensées de Léocadie Timothée quant à sa laideur sont directement verbalisées par les insultes de son entourage. Tout au long du texte, des chants, exclamations et autres prières permettent de sortir des pensées des personnages et de faire de l'œuvre un roman polyphonique donnant à lire différentes rumeurs populaires. L'importance de l'oralité et de la polyphonie dans Traversée de la Mangrove nous pousse à émettre quelques réserves quant à la filiation woolfienne que Richard Philcox voit chez Maryse Condé. On remarque à ce sujet que les différentes voix du roman ne sont pas autant mises en relief dans la traduction anglophone que dans l'œuvre originale. Le chant populaire sur Ti-Tor est typographiquement mis en relief par les espaces et les italiques chez Maryse Condé :

Quand Ti-Tor sétait installé, il y avait eu beaucoup de gens pour s'offusquer et chanter méchamment :

\section{Kouli malaba}

Isi dan

Pa peyiw

1. Coolie malabar (injurieux). Ce pays n’est pas le vôtre. (Maryse Condé 1989:20).

Par contre, dans la traduction de Richard Philcox, le chant constituant un discours détaché n'est pas typographiquement distingué du paragraphe constituant le récit principal : "When TiTor truned up, quite a few people took offense and chanted spitefully : "Kouli malaba isi dan pa peyiw !» (Coolie malar, this country’y not yours !). ( Maryse Condé 1995 : 8-9). Même le relief permis par la note de bas de page est effacé par le choix d'une traduction simultanée intégrée au corps du texte. Cette condensation des différentes voix du roman peut être reliée au parti pris de lauteur de ne faire entendre qu'un flot de pensées continues. Il y a ainsi une certaine interprétation personnelle de l'œuvre dans la version de Richard Philcox qui confère à Traversée de la Mangrove des allures woolfiennes.

Nous pouvons retenir de ce parcours de trois romans guadeloupéens que la traduction en contexte caribéen requiert lélaboration d'une stratégie transcriptrice ainsi qu'un positionnement 
du traducteur. Il s'agit pour le traducteur de faire des choix, de privilégier la fidélité à l'œuvre source et à ses particularités linguistiques au risque de complexifier la lecture dans la langue d'arrivée ou d’aménager quelque peu le texte pour permettre une lecture plus fluide de l’ouvre traduite. Certains tentent de trouver des compromis. Pensons à Clarisse Zimra qui n’a pas voulu laisser de trace de son intervention interprétative dans le corps du texte pour donner au lecteur la chance de le découvrir sous la forme la plus proche possible de l’œuvre source. Cela-dit, elle a laissé au lecteur frustré de n'avoir pas compris les subtilités de la langue de l'écrivain la possibilité de consulter son appareil critique à la fin de l'ouvrage. Marie-José Hoyet et Richard Philcox ont, quant à eux, mêlé plus directement leur aiguillage au corps du texte. Le traducteur de Maryse Condé propose même de s'affranchir du cadre caribéen avec sa traduction inspirée du style woolfien. Ce faisant, il rejoint les convictions de Maryse Condé signataire du manifeste « Pour une littérature-monde en français » revendiquant un déconfinement des imaginaires francophones.

Deux constantes sont à garder à l'esprit. Chaque texte est différent et nécessite une nouvelle approche traductive. De plus, le traducteur est tout d'abord un lecteur, à ce titre il exerce une lecture subjective et sa transposition variera en fonction des éléments auxquels il aura été le plus sensible dans le texte source. Sachant cela, il semble artificiel de mettre en place une hiérarchie qualitative des traductions. Étant sensible à la richesse plurilingue des romans caribéens, je suis particulièrement séduite par la démarche de Clarisse Zimbra qui tente de préserver au mieux la teneur plurilingue du roman de Daniel Maximin quelle traduit. Cette démarche est audacieuse dans le sens où elle prend le risque de perdre le lecteur las de devoir recourir au glossaire pour comprendre le sens de certains termes non expliqués dans le corps du texte. Il faut ainsi être reconnaissant envers la traductrice mais également envers léditeur qui a accepté de prendre ce risque avec elle.

\section{Références bibliographiques}

Barbery, M. ; Ben Jelloun, T. ; Borer A. ; Brival, R. ; \& Condé, M. et alii. (2007). Pour une littérature-monde en français. Le Monde, 15/03/2007. <http://www.lemonde.fr/livres/article/2007/03/15/des-ecrivainsplaident-pour-un-roman-en-francais-ouvert-sur-le-monde_883572_3260.html>

Chamoiseau, P. (1999). Solibo Magnificent. Trad. R.-M. Réjouis, \& V. Vinokurov. London : Granta Books.

- (1997). Texaco. Trad. R.-M. Réjouis, \& V. Vinokurov. New York : Vintage international. . (2001). De oude slaaf en de bloedhond. Trad. E. Van Hemert. Breda : De Geus.

Condé, M. (1989). Traversée de la Mangrove. Paris : Mercure de France.

(1995). Segu. Trad. B. Bray. New York : Ballantine Books.

(1995). Crossing the mangrove. Trad. R. Philcox. New York : Anchor Books.

Devoto, A. (2003). Traduire la littérature créole francophone : Chamoiseau, Confiant..., Table ronde animée

par Jean-Claude Lebrun. In Dix-neuvièmes assises de la traduction littéraire. Arles : Atlas, Actes Sud.

Fratta C. ; \& Torchi F. (2007). La littérature caribéenne francophone en Italie. In L. D’Hulst, J.-M. Moura, Li.

De Bleeker, \& Nadia Lie (Eds.). Caribbean Interfaces. Amsterdam: Rodopi.

Hoyet, M.-J. (Ed.) (2005). Voci dalle isole. Narrativa delle aree insulari francophone. Isernia : Cosimo Iannone. . (2005). Nigrizia : fatti e problemi del mondo nero : rivista mensile dei missionari comboniani,

123,4 . 
Hurbon, L. (2000). Le Phénomène religieux dans la Caraïbe : Guadeloupe, Martinique, Guyane, Haïti. Paris : Karthala.

Maximin, D. (1981). L'Isolé soleil. Paris : Seuil.

- (1987). Soufrières. Paris : Seuil. (1995). L'̂̂le et Une nuit. Paris : Seuil.

- (1989). Lone sun. Trad. C. Zimra. Charlottesville : The University Press of Virginia. . (2006). Les Fruits du cyclone : une géopoétique de la Caraïbe. Paris : Seuil.

Ndiaye, C. (2005). Transparence. In M. Beniamino, \& L. Gauvin (Eds.). Vocabulaire des études francophones : Les concepts de base. Paris : PULIM.

Pépin, E. (1992). L'Homme-au-Bâton. Paris : Gallimard.

- (1996). L'Uomo col bastone. Trad. M.-J. Hoyet. Roma: Edizione Lavoro. -. (2001). Africa solo, Éditions A3.

Schwarz-Bart, S. (1972). Pluie et vent sur Télumée Miracle. Paris : Seuil.

Stampfli, A. (2014). Peut-on traduire un écrit plurilinguistique sans le trahir ? Le cas des romanciers créolistes. La main de Thôt, 2. <http://revues.univ-tlse2.fr/lamaindethot/index.php?id=397> 